\title{
Quantum Dot in Z-shaped Graphene Nanoribbon
}

\author{
Z. F. Wang, ${ }^{1}$ Huaixiu Zheng, ${ }^{2}$ Q. W. Shi,, , Jie Chen, ${ }^{2}, 3$, 由 Qunxiang Li, ${ }^{1}$ and J. G. Hou ${ }^{1}$ \\ ${ }^{1}$ Hefei National Laboratory for Physical Sciences at Microscale \\ ${ }^{2}$ Electrical and Computer Engineering, University of Alberta, AB T6G 2V4, Canada \\ ${ }^{3}$ National Institute of Nanotechnology, Edmonton AB T6G 2V4, Canada
}

(Dated: November 1, 2018)

\begin{abstract}
Stimulated by recent advances in isolating graphene, we discovered that quantum dot can be trapped in Z-shaped graphene nanoribbon junciton. The topological structure of the junction can confine electronic states completely. By varying junction length, we can alter the spatial confinement and the number of discrete levels within the junction. In addition, quantum dot can be realized regardless of substrate induced static disorder or irregular edges of the junction. This device can be used to easily design quantum dot devices. This platform can also be used to design zero-dimensional functional nanoscale electronic devices using graphene ribbons.
\end{abstract}

PACS numbers: 73.61Wp, 61.72.Ji, 68.37.Ef

Due to its amazing electrical properties, carbon nanotubes (CNTs) discovered by Iijima [1] in 1991, have been considered as a leading candidate for nanoscale electronic applications. Major experimental and theoretical breakthroughs have been achieved [2, 3, 4], combining two distinct chirality carbon-nanotubes by introducing topological point defects in the graphene hexagonal lattice, to realize the quantum dot in the carbon nanotube heterojunctions. In such devices, the major sources of spin decoherence have been identified as the spin orbit interaction, coupling the spin to lattice vibrations and the hyperfine interaction of the electron spin with the surrounding nuclear spins. Therefore, it is desirable to form qubits in quantum dots based on these materials, where spin orbit coupling and hyperfine interaction are considerably weaker [5]. However, large scale integrated nanotube quantum dot devices are hard to make, because it is still difficult to assemble large numbers of CNTs together.

Very recently, the fabrication of a single layer of graphene and the measurement of its electric transport properties have been achieved [6, 7]. Graphene is a flat monolayer of carbon atoms tightly packed into a two-dimensional (2D) honeycomb lattice. What makes graphene so attractive for nanoelectronics is that the energy spectrum closely resembles the Dirac spectrum for massless fermions. For massless Dirac fermions, the band gap is zero and the linear dispersion law holds at low energy, quasiparticles in graphene behave differently from those in conventional metals and semiconductors, where the energy spectrum can be approximated by a parabolic dispersion relation. Research interest in this material has grown exponentially [6, 7, 8, 9].

As the building block of the carbon nanotube (CNTs), graphene can be viewed as a sheet of a unrolled singlewalled nanotube. Graphene has similar mechanical, thermal and electrical properties as those of CNTs. Unlike CNTs, its flat structure can be easily etched using conventional lithography techniques. The interconnection

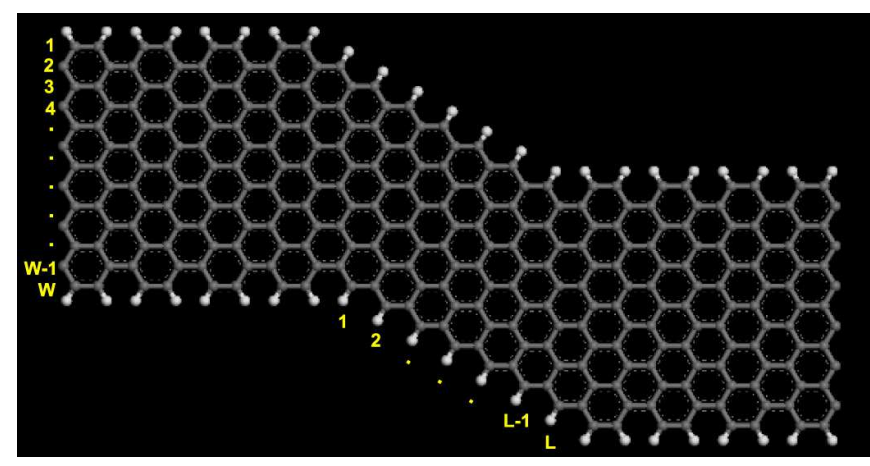

FIG. 1: (color online) Atomic structure of quantum-dot device made of perfect Z-shaped GNR junction. Width of the armchair GNR is labeled by integer W. Length of junction is labeled by integer L.

wires become unnecessary and integrated nanoelectronic devices can be completely made by using continuous graphene sheets. Armchair and zigzag graphene nanoribbons (GNR) are two basic graphene ribbon structures. Based on the nearest neighbour $\pi$ orbital tight binding model with diagonal matrix element fixed at Fermi level $\epsilon_{F}=0 \mathrm{eV}$ and all nonzero off diagonal matrix element set to $\gamma=-2.66 \mathrm{eV}$, zigzag GNRs are always metallic and the armchair GNRs are either metallic or semiconducting depending on its width $W$ 10]. For instance, when $W=3 n-1$, it is metallic, otherwise is semiconducting. Comparing with the recent Local density approximation (LDA) results [11, 12], except for the band gap opening for the ribbon with narrow width as the consequence of $\sigma$ bond length changing, GNR's electronic structure can still be quantitatively described using the simple tight binding model.

In addition to the study of 2-D and 1-D electronic proprieties of graphene, research attention has recently been focus on designing quantum dot (the 0 -D device) base on this novel material [13, 14, 15, 16]. In this letter, 
we propose a GNR quantum dot device, which consists of a $\mathrm{Z}$ shaped zigzag GNR junction connecting to two semi-infinite armchair GNRs as shown in Fig.1. Here, we suppose that GNR edge bonds are saturated by hydrogen atoms and no distortions exist in these GNRs. According to our numerical results, we find that this Zshaped junction device can completely confine electronic states induced by the topological structure of the junction. By varying the length of the junction, the spatial confinement and the number of discrete levels are modified accordingly. Surprisingly, these confined states can still exist even when considerable static disorders and irregular edges of the junction occur. This finding show that this quantum-dot device can be made without too many constraints, which indicates that such a device can be easily fabricated.

To study the electronic proprieties of the Z-shaped GNR junction, we separate the device as shown in Fig. 1 into three regions: the left lead, the middle junction, and the right lead. In this study, we assume that the junction width is $W-1$, the left/right leads have equal width $W$ and the length of the junction is $L$. Here $L$ and $W$ are integer. In this design, the leads are semi-infinite armchair GNRs, but the junction is a zigzag GNR. We have performed calculations using the nearest neighbour $\pi$ orbital tight binding model, the density of states (DOS) of the $\mathrm{Z}$ junction are determined by the direct diagonalization of $H=H_{c}+\Sigma_{L}^{r}+\Sigma_{R}^{r}$, where $H_{c}$ is the Hamilton of $\mathrm{Z}$ junction. Including parts of the armchair GNRs in the Hamilton of $H_{c}$ do not obviously change our results, so in the following calculation, only the zigzag junction part is included in the $H_{c}$. The contributions from the left and right leads are included in the self-energy term $\Sigma_{L / R}^{r}$, which are calculated using Green's function along with a transfer matrix technique [17, 18]. The DOS of this system can be expressed as $\operatorname{DOS}(E)=\sum_{\alpha} \frac{1}{2 \pi} \frac{\gamma_{\alpha}}{\left(E-\varepsilon_{\alpha}\right)^{2}+\left(\gamma_{\alpha}\right)^{2}}$. The summation covers all eigenvalues. $\varepsilon_{\alpha}$ is the real part of eigenvalue and represents the position of the state. $\gamma_{\alpha}$ is the imaginary part of the eigenvalue, which represents the broaden of the state. Local density of states (LDOS) are directly obtained from Green's function.

In Fig.2 (a), we show the DOS of a Z-shaped junction with $W=7$ and $L=4$. There are two sharp peaks in the energy range within the band gap. The position of the peaks is symmetric around $E=0 \mathrm{eV}$, because the topological structure of the junction does not break electron-hole symmetry. This phenomenon is dramatically different from the quantum dot in nanotube heterojunctions, in which the pentagon-heptagon interface break down this symmetry [2]. In Fig.2 (b), we show the spatial dependence of LDOS for the discrete states in a Z-shaped junction. In this case, the discrete energies are $E_{1}=-0.3 \mathrm{eV}$ and $E_{2}=0.3 \mathrm{eV}$. By considering energy symmetry, only the LDOS of $E_{1}=-0.3 \mathrm{eV}$ is plotted in Fig.2 (b). Since the LDOS corresponds to the
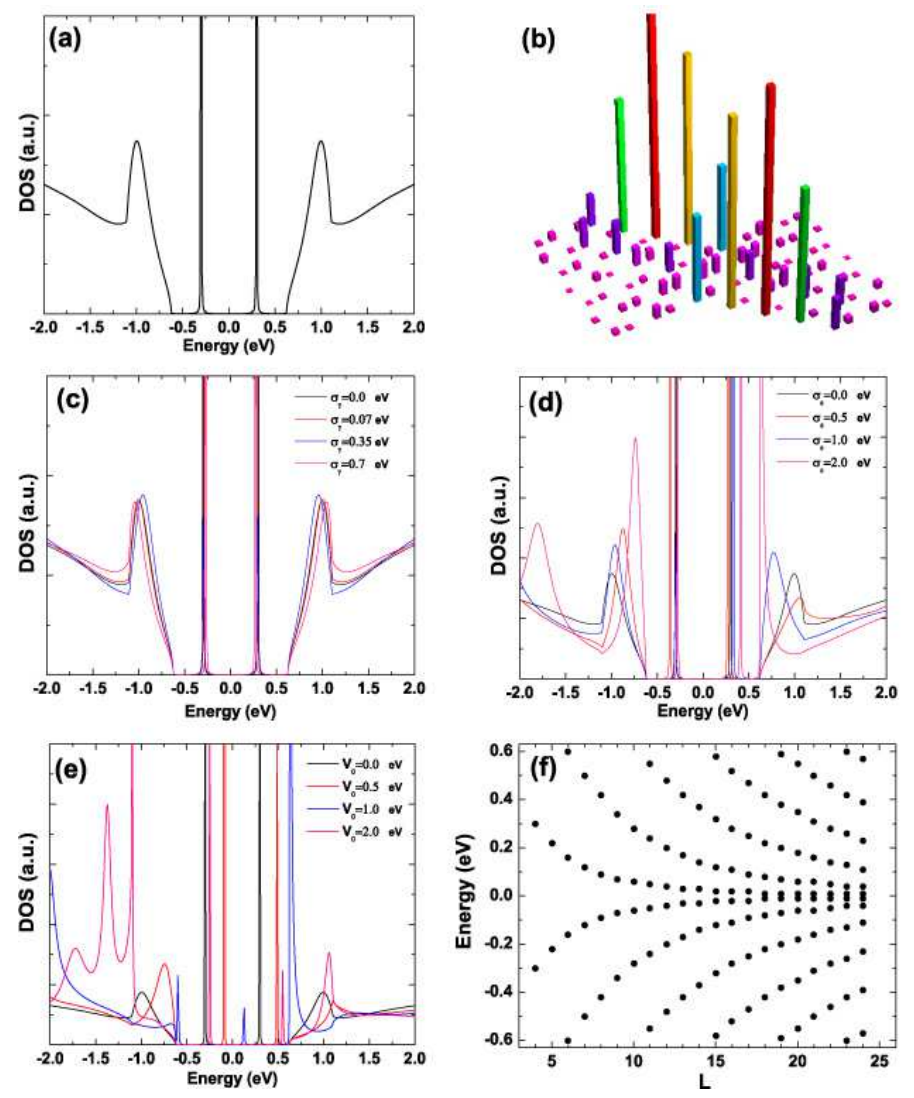

FIG. 2: (color online) Z-shaped junction with width $W=$ 7 and length $L=4$. (a) DOS of perfect junction without disorder. (b) The corresponding spacial distribution of LDOS of the perfect junction for $\mathrm{E}=-0.3 \mathrm{eV}$. (c) DOS of the junction when present short range disorder, $\sigma_{\gamma}$ changes with $0,0.07$, 0.35 and $0.7 \mathrm{eV}$. (d) DOS of the junction when present short range disorder, $\sigma_{\epsilon}$ changes with $0,0.5,1.0$ and $2.0 \mathrm{eV}$. (e) DOS of the junction when present long range disorder with one centered Gaussian potential $V(r)=V_{0} \exp \left(-r^{2} / 2 \sigma^{2}\right)$, sites on the lattice, here $\sigma=3.5 \AA$ and $V_{0}$ changes with $0,0.5,1.0$ and $2.0 \mathrm{eV}$. (f) Length dependence for the energy of confined states.

squared amplitude of the wave function, Fig.2 (b) illustrates the spatial localization of the $\mathrm{Z}$ shaped junction, i.e. the discrete states are localized in the junction region and especially along the edge of the junction. This phenomenon clearly demonstrates that these discrete states are caused by quantum confinement. The Z-junction design can be used as a quantum dot device. Unlike previous designs [13, 14, 15], quantum confinement in our study is due to the change in the network connectivity. These confined states are attributed to the surrounding barriers, which form by the interconnection between the armchair GNR and the zigzag GNR, which has been well used to explained the nanotube heterojunctions quantum $\operatorname{dot}$ 2, 3].

Perfect GNRs seldom exists in reality. Even if initial perfect, once physical adsorbed on a surface, GNRs will 
experience the effects of the disorder due to the interaction with the substrate [19]. This disorder can change their wave function and affect their usage in nanoelectronic devices. According to the potential range of the disorder, they can be classified into long-range and shortrange effects. The long-range effect (potential changes longer than the lattice distance) is caused by substrate charge and the short-range effect (potential varies rapidly at the scale of the lattice distance) is caused by residual interaction. The effect of these disorders can be simulated by adjusting corresponding elements in the Hamilton matrix [19]. For instance, the short-range disorder effect can be modeled by assuming that the diagonal and the nonezero off diagonal matrix elements independently fluctuate around their initial values with variances $\sigma_{\epsilon}$ for diagonal elements and $\sigma_{\gamma}$ for nonzero off diagonal elements. The long range disorder can be stimulated by introducing a 2D Gaussian potential with the form of $V(r)=V_{0} \exp \left(-r^{2} / 2 \sigma^{2}\right)$ centered around the carbon site to shift the diagonal element. In the following calculation, the disorder is limited in the junction region for simplicity.

The effects of the substrate induced static disorder to the confined states in the Z-shaped junction are shown in Fig.2 (c),(d) and (e). To see the disorder effects more clearly, we consider only one type of disorders in Fig.2 (c) $\sim(\mathrm{e})$, respectively. In Fig.2 (c), we suppose that only the short-range disorder with $\sigma_{\gamma}$ exists within the junction region. Considering the $\mathrm{C}-\mathrm{C}$ bond of length $d$, the corresponding nonzero off diagonal element $\gamma$ will change $\delta \gamma=\alpha \delta d$ with $\alpha \simeq 47 \mathrm{eV} / \mathrm{nm}$ [20]. So in Fig. 2(c), for $\sigma_{\gamma}=0.07,0.35$ and $0.7 \mathrm{eV}$, the $\mathrm{C}-\mathrm{C}$ bond lengths change as much as $\pm 1 \%, \pm 5 \%$ and $\pm 10 \%$. Even the bond length changes $10 \%$. The DOS, however, does not change much except for a slight peak shift of the discrete states. This off-diagonal short-range disorder has almost no impact to the confined states. In addition, the DOS remains symmetric around $E=0 \mathrm{eV}$, which indicates that this type of disorder cannot break the electron-hole symmetry in this junction device. Secondly, in Fig.2 (d), we only consider the short-range disorder $\sigma_{\epsilon}$. With different disorder strengths as much as $\sigma_{\epsilon}=0.5,1.0$ and $2.0 \mathrm{eV}$, fortunately, these confined states can still exist. Unlike the former case, the position of the confined states change more dramatically for the $\sigma_{\epsilon}$ disorder and they are not no longer symmetric around $E=0 e V$. Lastly, for the long-range disorder, a Gaussian potential is introduced in the junction range. The shift of different potential center, however, will not change our findings (we only plot one of them in Fig.2 (d) for illustration). Compare to the $\sigma_{\epsilon}$ disorder, the DOS becomes more asymmetric around $E=0 e V$. Two confined states, however, still exist within the gap region. These phenomena can be easily explained, because the discrete states are confined by the two barriers formed by the change in the network connectivity. The substrate induced disorder cannot change the topological barriers much, and thus it still has enough strength to confine the electron in the junction and form a quantum dot.
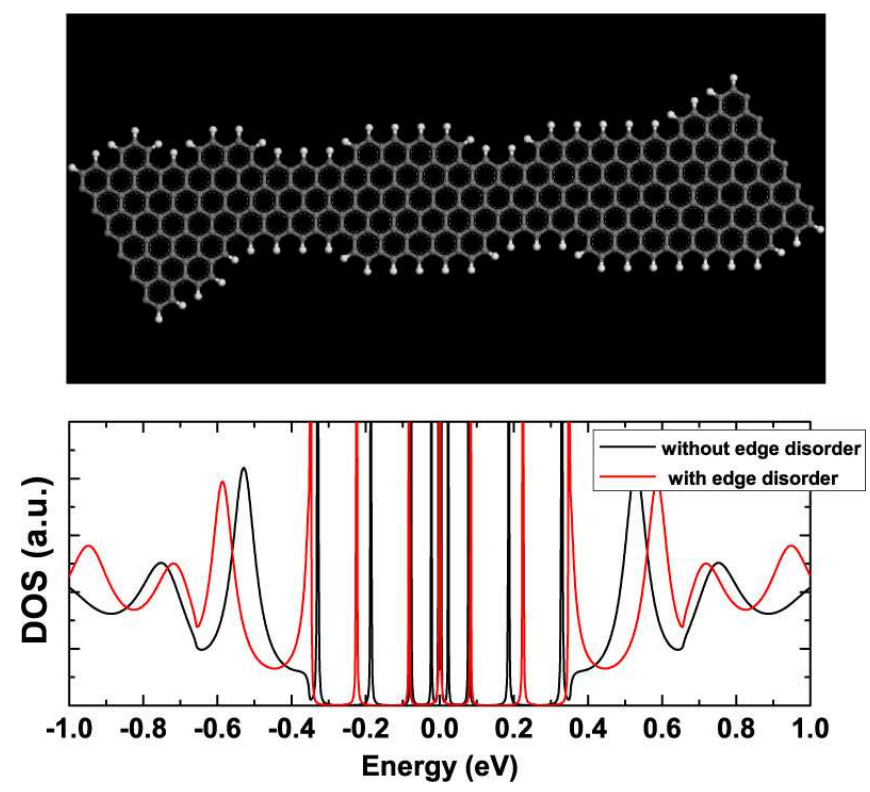

FIG. 3: (color online) Top figure: the atomic structure of a Z-shaped junction of $W=13$ and $L=20$ with irregular edge. Bottom figure: The DOS of a perfect $\mathrm{Z}$ junciton and an irregular edge $\mathrm{Z}$ junction.

To extend this novel quantum-dot design, we also investigate how the junction length affects quantum-dot confinement. The length of the quantum-dot along the junction is $L \times 2.13 \AA$. Here we assume no disorder exists in the junction region, Fig.2 (f) shows the energy of the confined states vs. the length of the quantum-dot. As expected, when $L$ increases, the number of confined states increases as well. Furthermore, with the increasing length of this quantum-dot device, the confined states around $E=0 e V$ are almost degenerated. In addition, within in the range of the $\mathrm{L}$ value studied, the energy spacing between the discrete level is about $\simeq 100 \mathrm{meV}$ except for these states around $E=0 e V$. This value is larger than the thermal broadening at room temperature.

Unlike CNT devices, GNR device most likely have irregular edges due to lithography. Finally in our study, we consider the impact of edge disorder in zigzag junction region. Here, we follows the method suggested by Areshkin to simulate eroded zigzag edge [19]. This method ensure that all the $\mathrm{C}$ atoms remains 3 -fold coordinated with at most one $\mathrm{H}$ atom attached to an edge $\mathrm{C}$ atom without steric problem. Here we restrict the erosion to the outmost layer of the edge. Fig.3 shows how these defects affect the confined states. Similar to the case of the $\sigma_{\gamma}$ disorder, a slight peak shift can be observed. However, the confined states still exist even under the presence of the irregular edges. We can explain it in a straight 
way. This edge disorder erodes some carbon and hydrogen atoms in the zigzag junction. The effects of breaking bonds are equivalent to add $-\gamma$ to the nonzero off diagonal elements in the Hamilton matrix similar to the $\sigma_{\gamma}$ disorder. The effects for eroded hydrogen can be neglected because carbon atoms can form strong covalent bonds with hydrogen atom. The $\sigma$ bands of these bonds lie far away from the Fermi surface and do not need to be considered. This result is extremely important and provides more convenience and flexibility to implement this structure in experiments. With the increasing width of the GNR junction, the ribbon behaviors will gradually transit to that in a $2 \mathrm{D}$ graphene. The edge effects would become much weaker. Therefore, a wider GNR junction should be able to tolerate even a greater degree of edge disorder. However, these imperfections do not change the behavior of our quantum-dot device, which is very important in future nanofabrication.

In summary, we propose a quantum-dot device design using a Z-shaped GNR junction. This system can completely confine electronic states induced by the topological structure of the Z-junction. By varying the length of this junction device, the spatial confinement and the number of discrete levels can be modified. In addition, the substrate induced static disorder and the irregular edges of GNRs do not destroy these confined states. These findings provide a convenient way to fabricate this structure experimentally. For our on-going study, we are investigating the effect of coulomb interactions in this structure. Overall, our contribution is to provide a simple model to design zero-dimensional functional nanoscale electronic devices using graphene ribbons.

This work is partially supported by the National Natural Science Foundation of China with grant numbers 10574119, 10674121 and 50121202. The research is also supported by National Key Basic Research Program under Grant No. 2006CB922000, Jie Chen would like to acknowledge the funding support from the Discovery program of Natural Sciences and Engineering Research Council of Canada under Grant No. 245680.

* Author to whom correspondence should be addressed. Electronic address:phsqw@ustc.edu.cn

$\dagger$ Author to whom correspondence should be addressed. Electronic address jchen@ece.ualberta.ca

[1] S. Iijima, Nature 354, 56 (1991).

[2] L. Chico, M. P. L'ez Sancho and M. C. Muñz, Phys. Rev.
Lett. 81, 1278 (1998).

[3] C. G. Rocha, T. G. Dargam, and A. Latgé, Phys. Rev. B 65, 165431 (2002).

[4] Yagang Yao, Qingwen Li, Jin Zhang, Ran Liu, Liying Jiao, Yuntian T. Zhu, and Zhongfan Liu, Nature Materials 6,293 (2007).

[5] Hongki Min, J. E. Hill, N. A. Sinitsyn, B. R. Sahu, Leonard Kleinman, and A. H. MacDonald, Phys. Rev. B 74, 165310 (2006).

[6] K. S. Novoselov, A. K. Geim, S. V. Morozov, D. Jiang, M. I. Katsnelson, I. V. Grigorieva, S. V. Dubonos, and A. A. Firsov, Nature 438,197 (2005).

[7] Claire Berger, Zhimin Song, Xuebin Li, Xiaosong Wu, Nate Brown, Cécile Naud, Didier Mayou, Tianbo Li, Joanna Hass, Alexei N. Marchenkov, Edward H. Conrad, Phillip N. First and Walt A. de Heer, Science 312,1191 (2006).

[8] Sasha Stankovich, Dmitriy A. Dikin1, Geoffrey H. B. Dommett, Kevin M. Kohlhaas, Eric J. Zimney, Eric A. Stach, Richard D. Piner, SonBinh T. Nguyen and Rodney S. Ruoff, Nature 442, 282 (2006); Jannik C. Meyer, A. K. Geim, M. I. Katsnelson, K. S. Novoselov, T. J. Booth and S. Roth, Nature 446, 60 (2007); Hubert B. Heersche, Pablo Jarillo-Herrero, Jeroen B. Oostinga, Lieven M. K. Vandersypen and Alberto F. Morpurgo, Nature 446, 56 (2007).

[9] Z. F. Wang, Ruoxi Xiang, Q. W. Shi, Jinlong Yang, Xiaoping Wang, J. G. Hou, and Jie Chen, Phys. Rev. B 74, 125417 (2006); Z. F. Wang, Qunxiang Li, Haibin Su, Xiaoping Wang, Q. W. Shi, Jie Chen, Jinlong Yang, and J. G. Hou, Phys. Rev. B 75, 085424 (2007); Z. F. Wang, Qunxiang Li, Huaixiu Zheng, Hao Ren, Haibin $\mathrm{Su}, \mathrm{Q}$. W. Shi, and Jie Chen, Phys. Rev. B 75, 113406 (2007).

[10] Kyoko Nakada, Mitsutaka Fujita, Gene Dresselhaus and Mildred S. Dresselhaus, Phys. Rev. B 54, 17954 (1996).

[11] Y. Son, M. L. Cohen and S. G. Louie, Nature 444,347 (2006).

[12] Y. Son, M. L. Cohen and S. G. Louie, Phys. Rev. Lett. 97, 216803 (2006).

[13] Björn Trauzettel, Denis V. Bulaev, Daniel Loss and Guido Burkard, Nature Physics 3,192 (2007).

[14] J. Milton Pereira, Jr., P. Vasilopoulos, and F. M. Peeters, Nano Lett 0,4 (2007).

[15] A. Rycerz, J. Tworzyd and C. W. J. Beenakker, Nature Physics 3,172 (2007).

[16] P. G. Silvestrov and K. B. Efetov, Phys. Rev. Lett. 98, 016802 (2007).

[17] M P López Sancho, J M López Sancho and J Rubio, J. Phys. F : Met. Phys. 15, 851 (1985).

[18] S. Datta, Electronic Transport in Mesoscopic Systems (Cambridge University Press, Cambridge, England, 1995).

[19] Denis A. Areshkin, Daniel Gunlycke and Carter T. White, Nano Lett 7,204 (2007).

[20] C. T. White and T. N. Todorov, Nature 393,240 (1998). 